\title{
Difficulties and challenges to diagnose and treat post-traumatic long bone osteomyelitis
}

\author{
M. E. Hake • J. K. Oh $\cdot$ J. W. Kim • \\ B. Ziran - W. Smith · David Hak · C. Mauffrey
}

Received: 1 December 2014/ Accepted: 2 December 2014/Published online: 6 December 2014

(C) Springer-Verlag France 2014

\section{Background}

The management of post-traumatic long bone osteomyelitis remains challenging [1]. While significant progress has been made over the past 10-20 years, many simple questions such as optimal duration or route of antibiotics administration remain unanswered [2]. General principles of early aggressive management, optimization of host factors and wide debridement of affected tissues with later reconstruction are commonly accepted. These principles share similarities with those applied in tumor surgery [3]. Unfortunately, specific diagnostic and treatment strategies are ill-defined and lack prospective evidence. In this paper, we highlight some of the limitations that remain regarding the prevention, diagnosis and treatment of post-traumatic long bone osteomyelitis.

M. E. Hake · J. W. Kim · D. Hak · C. Mauffrey $(\bowtie)$

The Department of Orthopaedics, Denver Health Medical Center, University of Colorado School of Medicine, 777 Bannock Street, Denver, CO 80204, USA

e-mail: cyril.mauffrey@dhha.org; cmauffrey@yahoo.com

J. K. Oh

The Department of Orthopaedics, Korea University Guro Hospital, Seoul, Korea

B. Ziran

The Department of Orthopaedics, The Hughston Clinic at Gwinnett Medical Center, Atlanta, GA, USA

W. Smith

The Department of Orthopaedic Surgery, Swedish Medical Center, Englewood, CO 80113, USA

\section{Numerous variables in the disease and host type}

Post-traumatic long bone osteomyelitis is a heterogeneous condition or a spectrum of disease. In fact, the location, severity of the infection and type of host can vary significantly, making consistency among study cohorts difficult. The excellent blood supply and good soft tissue coverage of the upper extremity contribute to lower infection rates, higher success rates and different treatment modalities than lower extremity bony infections [4]. Treatment strategies that include use of antibiotic-loaded cements or antibiotic beads are not FDA approved and therefore cannot be studied prospectively in the USA. This is a significant roadblock in the prospective evaluation of the safety and efficacy of these modalities.

\section{What clinically relevant end points should be selected?}

Effective treatment requires that realistic goals be established with well-defined end point and that the patients understand and accept these goals. In some cases, eradication of the infection may only be obtained by aggressive resection of a long bone or intra-articular segment, at the expense of patient's functional outcome. On the other end of the spectrum, a return to baseline function may be acceptable with the requirement of longterm suppressive antibiotics and multiple procedures. As cost-effectiveness becomes more of a focus in today's healthcare environment, the long-term expense of limb salvage versus amputation will need consideration in advanced cases or those requiring complex and lengthy reconstruction procedures. With a greater emphasis on quality care and patient satisfaction, it is challenging to establish the role these factors play in the selection of an 
appropriate and individualized diagnostic and treatment strategy.

\section{Which diagnostic strategies are reliable?}

The first challenge in treating a patient with possible osteomyelitis is to establish a diagnosis. Clinical examination is critical with the presence of a draining sinus, pain, redness and swelling, being findings that can confirm the presence of a deep infection. Laboratory markers lack sensitivity to be used in isolation $[5,6]$. Radiologic tools, such as plain radiographs and MRI, cannot make a definitive diagnosis, and MRI is often of little use when metallic implants are involved.

The gold standard remains a bone culture, but falsenegative rates as high as $40 \%$ are reported. This high rate may be attributed to sampling errors and/or presence of biofilm [7]. It is therefore crucial to combine the abovementioned tools to establish a diagnosis. Specimens harvested and sent for microbiology should also be sent to pathology for histological confirmation (especially bone tissue). In addition, biopsies should be taken from different sites (at least 4 ) to reduce sampling errors.

The Cierny and Mader classification [8], which correlates with treatment strategies, may be arduous to establish. The host component of this classification scheme may be the most important predictor of outcomes and less prone to inter observer variations; however, accurate determination of the stage (medullary, localized, superficial or diffuse) can be complex and may require MRI to evaluate the extent of the disease. We have concerns regarding over-reliance on MRI as the majority of patients with post-traumatic osteomyelitis have metallic implants in place, making interpretation of MRI images difficult at best. In addition, one of the major pitfalls of the current classification schemes is that it does not take into account the anatomical location in the long bone (epiphyseal, metaphyseal or diaphyseal). This location, in our opinion, will have dramatic impact on the selection of treatment strategies.

The role of molecular biology for the diagnosis of posttraumatic long bone osteomyelitis remains to be confirmed as an alternative with the growing concerns of biofilm as a possible cause of high false-negative rates in standard cultured tissues [9]. The usefulness of sonication, polymerase chain reaction (PCR) and fluorescent in situ hybridization (FISH) to confirm or diagnose osteomyelitis has only recently begun to be evaluated [10]; however, our current expert opinion recommends a combination of history, physical, laboratory testing (ESR, CRP) and biopsy as a reasonable standard for evaluating osteomyelitis. Addition studies such as MRI and nuclear-tagged white blood cells scans may be useful in addition, but should never supplant the above standard recommendations.

\section{Treatment strategies}

Once diagnosis is confirmed, it is critical to have a discussion with the patient about treatment expectations. Unlike a fractured bone where the expectations are implicitly defined, those for the management of a tibial osteomyelitis can lack clarity. Expectations from the surgeon may be different from those of the patient and their relatives. Choosing whether to operate for infection eradication or suppression is a big decision that can have a huge impact on the future of the patient. In many cases, extirpation of an infection will require amputation, while suppression will not.

An additional challenge in choosing treatments and prognosticating outcome is the imperfection of our tools to measure infection eradication. For deep-seated diffuse infections that require debridement and reconstructions, we still rely upon the "paprika sign" to determine intraoperative margins of debridement [11, 12].

Quantification of success of a surgery is often times as difficult as making the initial diagnosis. C-reactive protein and erythrocyte sedimentation rates can be unreliable, especially in the immune-compromised patient [13]. Postoperative MRI is not useful when metallic implants are in the area of concern; however, modern MRI may be able to provide more accurate information despite the presence of hardware. MRI-compatible implants such as carbon fiber nails to stabilize and reconstruct segmental defects may have a valid role in the future [14].

Our ability to predict cure, short of radical resection, is imperfect, and we must communicate this to the patient in the planning process [15].

\section{The future}

Preventative measures must be studied to reduce the incidence of post-traumatic long bone infection. The role of antibiotic-coated implants is being evaluated [16]. Our research team is currently investigating biofilm formation and its affinity to various types of implants including antibiotic cement-coated nails. Our hypothesis is that biofilm formation has an affinity that varies based on the specific implanted material. This remains to be confirmed in an animal open fracture model.

Research on novel diagnostic strategies is under way, especially with the development of more accurate technologies utilizing molecular biology tools. PET scans 
remain expensive, but may become a great addition to our diagnosis armamentarium.

The treatment of post-traumatic osteomyelitis is illdefined, and we lack an algorithm based on the location in bone, host type, physical findings and patients' expectations. The development of novel tools such as the reamer irrigator aspirator (RIA) both to harvest tissue samples and to debride infected intramedullary cavity has changed the way that some of us manage such complex infections [17]. Finally, more research should be performed on specific patient-derived outcome measures that may be applied to patients suffering with long bone osteomyelitis.

\section{Take-home message}

Post-traumatic long bone osteomyelitis remains a challenging condition to diagnose and treat. Further research should be directed at a classification system that can direct treatment, diagnostic methods with high specificity and treatment algorithms based on specific patients' expectations.

The field of post-traumatic infections lags behind the arthroplasty literature by several years. The orthopedic trauma community must start collaborating to increase the power of the data that we currently have and develop more robust treatment algorithms. The idea of expert consensus with future directions for research should also be explored.

Conflict of interest Dr. C. Mauffrey's institution was recipient of a research grant from Carbofix.

\section{References}

1. Lew DP, Waldvogel FA (1997) Osteomyelitis. N Engl J Med 336(14):999-1007

2. Sanders J, Mauffrey C (2013) Long bone osteomyelitis in adults: fundamental concepts and current techniques. Orthopedics 36(5):368-375

3. Lazzarini L, Mader JT, Calhoun JH (2004) Osteomyelitis in long bones. J Bone Joint Surg Am 86(10):2305-2318
4. Honda H, Mcdonald JR (2009) Current recommendations in the management of osteomyelitis of the hand and wrist. J Hand Surg Am 34(6):1135-1136

5. Parvizi J, Gehrke T, Chen AF (2013) Proceedings of the international consensus on periprosthetic. Joint Infection Bone Joint $\mathrm{J}$ 95(11):1450-1452

6. Carragee EJ, Kim D, Van der Vlugt T, Vittum D (1997) The clinical use of erythrocyte sedimentation rate in pyogenic vertebral osteomyelitis. Spine 22(18):2089-2093

7. Costerton JW (2005) Biofilm theory can guide the treatment of device-related orthopaedic infections. Clin Orthop Relat Res 437:7-11

8. Cierny G, Mader JT, Penninck JJ (2003) A clinical staging system for adult osteomyelitis. Clin Orthop Relat Res 414:7-24

9. Costerton JW, Stewart PS, Greenberg EP (1999) Bacterial biofilms: a common cause of persistent infections. Science 284(5418):1318-1322

10. Palmer MP, Altman DT, Altman GT et al (2014) Can we trust intraoperative culture results in nonunions? J Orthop Trauma 28(7):384-390

11. Simpson AH, Deakin M, Latham JM (2001) Chronic osteomyelitis. The effect of the extent of surgical resection on infectionfree survival. J Bone Joint Surg Br 83(3):403-407

12. Gokalp MA, Guner S, Ceylan MF, Doğan A, Sebik A (2014) Results of treatment of chronic osteomyelitis by gutter procedure and muscle flap transposition operation. Eur J Orthop Surg Traumatol 24(3):415-419

13. Forsberg JA, Potter BK, Cierny G, Webb L (2011) Diagnosis and management of chronic infection. J Am Acad Orthop Surg 19(Suppl 1):S8-S19

14. Mauffrey C, Chaus GW, Butler N, Young H (2014) MR-compatible antibiotic interlocked nail fabrication for the management of long bone infections: first case report of a new technique. Patient Saf Surg 8(1):14

15. Ferry T, Valour F, Lustig S, Laurent F, Perrin G, Chidiac C, Barrey C (2013) The challenge of infection prevention in spine surgery: an update. Eur J Orthop Surg Traumatol 23(Suppl 1):S15-S19

16. Plocki SC, Armbruster D, Klein K, Kämpf K, Zlinszky K, Hilbe M, Kronen P, Gruskin E, von Rechenberg B (2012) Biodegradable sleeves for metal implants to prevent implant-associated infection: an experimental in vivo study in sheep. Vet Surg 41:410-421

17. Kanakaris N, Gudipati S, Tosounidis T, Harwood P, Britten S, Giannoudis PV (2014) The treatment of intramedullary osteomyelitis of the femur and tibia using the reamer-irrigator-aspirator system and antibiotic cement rods. Bone Joint J 96(6):783-788 